\title{
PULSAR/SUPERNOVA REMNANT ASSOCIATIONS
}

\author{
D. A. FRAIL \\ National Radio Astronomy Observatory \\ P.O. Box 0, Socorro, NM, 87801, USA
}

\section{Introduction}

If one is interested in issues related to the birth and evolution of neutron stars, a promising avenue of research begins with the study of the byproducts of a Type II supernova event, namely a pulsar (PSR) and its supernova remnant (SNR). However, it has long been a matter of some embarrassment that out of the more than 600 known PSRs and the 175 catalogued SNRs there are few associations. In fact, for nearly 15 years following the discovery of PSRs only the Crab and Vela PSRs had an associated remnant. This failure has spawned something of a cottage industry to "explain away" the low incidence of PSR/SNR associations. Beaming (Frail \& Moffett 1993; Narayan \& Schaudt 1988), injection (Narayan 1987), field growth (Blandford, Applegate \& Hernquist 1983) and black-hole formation (Chevalier 1994) have been invoked in this context at one time or another.

In the last decade there has been tremendous progress in this field. These advances have come from two approaches: (1) High-frequency PSR searches of the Galactic plane (e.g., Johnston et al. 1992) which have overcome the high brightness temperatures and interstellar scattering that plagued previous surveys, allowing us to see deeper into the plane of the Galaxy, reaching the young, luminous PSRs, and (2) radio imaging of the fields of these young PSRS using the latest in low-frequency imaging algorithms (Cornwell 1993) has resulted in the discovery of extended, low surface brightness SNRs (e.g., Kassim \& Weiler 1990).

The last comprehensive review on PSR/SNR associations was by Helfand \& Becker (1984). Since then the incremental progress in this area has been reviewed by Kulkarni (1990) and Bailes \& Johnston (1993). In the spirit of these later mini-reviews we will describe a number of recent PSR/SNR associations and summarize some preliminary conclusions reached from a study of all the PSR/SNR associations by Frail, Goss \& Whiteoak (1994). 


\section{Pulsar-Supernova Remnant Associations}

\subsection{PSR 1338-62 AND G 308.8-0.1}

This PSR/SNR pair is a good illustration of how improvements in both image fidelity and PSR parameters have led to new associations. Both the PSR (Manchester, D'Amico \& Tuohy 1985) and the SNR (Caswell, Milne \& Wellington 1981) had been previously known but the association was not proposed until Caswell et al. (1992) made a sensitive radio image of G 308.8-0.1 and Kaspi et al. (1992) made timing observations of the PSR to refine the position of the PSR and establish its young age $(12 \mathrm{kyrs})$. The near central location of the PSR within G 308.8-0.1, and the close agreement in the ages argue persuasively for a real association.

Other recent examples where improved imaging has led to newly proposed associations of known PSRs include PSR 1757-24 and G 5.4-1.2 (Frail \& Kulkarni 1991), PSR 1800-21 and W 30 (Kassim \& Weiler 1990), PSR 170644 and G 343.1-2.3 (McAdam, Osborne, \& Parkinson 1993).

\subsection{PSR 1643-43 AND G $341.2+0.9$}

PSR 1643-43 has a period $P$ of $232 \mathrm{msec}$, a characteristic age $\tau_{\mathrm{c}}$ of $32.6 \mathrm{kyrs}$ and a dispersion measure based distance of $6.9 \mathrm{kpc}$ (Johnston et al. 1994). Recent work by Frail et al. (1994) shows that PSR 1643-43 lies in an extended $\left(22^{\prime} \times 16^{\prime}\right)$, non-thermal radio source which is likely a supernova remnant G $341.2+0.9$. The $\Sigma$ - $D$ distance of G $341.2+0.9$ is between 8.3$9.7 \mathrm{kpc}$ (Milne 1979; Clark \& Caswell 1976; Allakhverdiyev et al. 1983). In addition to the shell-type emission which brightens on the side closest to PSR 1643-43, there is diffuse emission in the immediate vicinity of the PSR. This includes a $4^{\prime}$ nebulosity just east of the PSR which is joined to the PSR by a "bridge" of emission. The appearance of this structure is consistent with an implied westward motion of the PSR.

Frail et al. (1994) argue that the similar distances of the PSR and the SNR in combination with the morphological evidence, are strong indicators that a real physical association exists between the two. Other PSR/SNR associations studied by Frail et al. (1994) include PSR 1706-44/G 343.1-2.3 and PSR 1727-33/G 354.1+0.1.

\section{The Distribution of Pulsar Velocities at Birth}

All known PSRs with characteristic ages less than 60,000 years are included in the table below. Of the 18 PSRs in this table there are 15 potential $\mathrm{PSR} / \mathrm{SNR}$ associations. One of the most striking characteristics of these associations is that in the majority of cases the PSR is offset from the center of its SNR. Following Shull et al. (1989) we quantify this by the 
parameter $\beta=\theta_{\mathrm{p}} / \theta_{\mathrm{s}}$ where $\theta_{\mathrm{p}}$ is the angular displacement of the PSR from the geometric center of the remnant, $\theta_{\mathrm{s}}$ is the angular radius of the supernova remnant. Note the values of $\beta$ in the table are rarely close to zero, and in fact in a few cases $\beta>1.0$ (i.e., the PSR is outside the remnant). If the PSR were born at the center of the remnant then we can straightforwardly calculate an implied PSR velocity $V_{\mathrm{PSR}}$ by dividing its transverse displacement from the center of the remnant by its age $\tau_{\mathrm{c}}$.

The median of these transverse velocities is $480 \mathrm{~km} \mathrm{~s}^{-1}$. Twelve of the 15 young PSRs have velocities above the mean of $217 \mathrm{~km} \mathrm{~s}^{-1}$ for the Harrison et al. (1993) proper-motion sample. Such large $V_{\mathrm{PSR}}$ values have been noted before in discussions of individual associations (e.g., Kassim \& Weiler 1990; Manchester et al. 1991), but it has only recently been recognized (Frail et al. 1994; Caraveo 1993) that this may be a general property of young PSRs as a whole. In an independent study, Lyne \& Lorimer (1994) corrected for well-known selection effects in proper-motion samples (Cordes 1986; Helfand \& Tademaru 1977) and used the newer Taylor \& Cordes (1993) distance model to derive a mean PSR birth velocity of $450 \pm 90 \mathrm{~km} \mathrm{~s}^{-1}$.

TABLE 1. Young Pulsars and Proposed Associated Supernova Remnants

\begin{tabular}{cccccrr}
\hline $\begin{array}{c}\text { PSR } \\
\text { Name }\end{array}$ & $\begin{array}{c}P \\
(\mathrm{~s})\end{array}$ & $\begin{array}{c}D \\
(\mathrm{kpc})\end{array}$ & $\begin{array}{c}\text { Age } \\
\left(10^{3} \mathrm{yrs}\right)\end{array}$ & $\begin{array}{c}\text { SNR } \\
\text { Name }\end{array}$ & $\beta$ & $\begin{array}{c}V_{\mathrm{PSR}} \\
\left(\mathrm{km} \mathrm{s}^{-1}\right)\end{array}$ \\
\hline $0531+21$ & 0.033 & 2.0 & 1.3 & Crab Nebula & & 150 \\
$1509-58$ & 0.150 & 4.4 & 1.5 & MSH 15-52 & 0.25 & 3100 \\
$0540-69$ & 0.050 & 49.4 & 1.7 & SNR 0540-693 & 0.06 & 260 \\
$1610-50$ & 0.232 & 7.3 & 7.4 & Kes 32 & 1.7 & 3600 \\
$0833-45$ & 0.089 & 0.5 & 11 & Vela XYZ & 0.02 & 120 \\
$1338-62$ & 0.193 & 8.7 & 12 & G 308.8-0.1 & 0.30 & 750 \\
$1757-24$ & 0.125 & 4.6 & 15 & G 5.4-1.2 & 1.3 & 1800 \\
$1800-21$ & 0.134 & 3.9 & 16 & W 30 & 0.73 & 1100 \\
$1706-44$ & 0.102 & 1.8 & 17 & G 343.1-2.3 & 1.4 & 670 \\
$1853+01$ & 0.267 & 3.3 & 20 & W 44 & 0.58 & 420 \\
$1046-58$ & 0.124 & 3.0 & 20 & & & \\
$1737-30$ & 0.607 & 3.3 & 21 & & & \\
$1823-13$ & 0.101 & 4.3 & 21 & & & \\
$1727-33$ & 0.139 & 4.2 & 26 & G 354.1+0.1 & & 460 \\
$1643-43$ & 0.232 & 6.9 & 32 & G 341.2+0.9 & 0.73 & 480 \\
$1930+22$ & 0.144 & 9.8 & 40 & G 57.1+1.7 & 0.45 & 1600 \\
$2334+61$ & 0.495 & 2.5 & 41 & G 114.3+0.3 & 0.0 & $<50$ \\
$1758-23$ & 0.416 & 3.0 & 59 & W 28 & 1.3 & 300 \\
\hline
\end{tabular}




\subsection{A CAVEAT OR TWO}

There are some potential difficulties with this method of determining PSR velocities from their offsets from the SNR center.

(1) Not all the associations in the table are secure and much effort needs to be made to test whether these associations are real or not. However, eliminating questionable associations does not significantly change the result (Frail et al. 1994). We also know that only a few percent of the disk is covered by SNRs and thus chance superpositions are rare. $85 \%$ of the PSRs in the table are found in or next to an SNR, compared with only $3 \%$ for PSRs with $\tau_{\mathrm{c}}>10^{5} \mathrm{yrs}$. Furthermore, there is no indication that we are searching any deeper around young PSRs than in general purpose low frequency interferometric surveys of the Galactic plane (e.g., Taylor, Wallace \& Goss 1992).

To properly demonstrate that a PSR/SNR association is a true physical association and not just a chance superposition of objects on the sky, agreement is required between the ages and distances of both objects. For the most part the distances and ages for the PSRs and SNRs in the table agree but they are poorly known, reflecting the uncertainty in the methods that are being used (H I absorption, DM distance, $\Sigma$-D, Sedov ages, etc.). Morphological evidence, such as a flat spectrum plerionic nebula surrounding the PSR or signs of increased brightness in the radio SNR shell closest to the PSR, suggests an interaction and hence is useful. Finally, a measured PSR proper motion is a useful and final discriminant for distinguishing real and chance superpositions. Unfortunately, Crab and Vela are the only PSRs in the table to have had such measurements made and as such they represent the most firm associations.

(2) This method rests on the assumption that we can identify the birthplace of the PSR as the center of the SNR. This issue is discussed in more depth by Frail et al. (1994). In short, it is necessary to have a high quality image of the SNR before determining PSR velocities by this method.

The Vela association is a good illustration of the dangers of using this method when the shape of the SNR is poorly known. Based on an offset of the PSR from the center of the Vela SNR the inferred proper motion of the PSR was thought to be $800 \mathrm{~km} \mathrm{~s}^{-1}$ and yet proper-motion measurements yielded a considerably lower value of $120 \mathrm{~km} \mathrm{~s}^{-1}$ (Bailes et al. 1989). However, the true shape of the remnant has only recently been defined by low-frequency radio observations (Dwarakanath 1991) and X-ray observations (Aschenbach 1992) revealing a larger and more circular remnant than had been previously suspected. Now if we use the new center of the remnant the inferred velocity of the PSR is the same as its proper motion value. 


\section{Implications}

The implications of the existence of large numbers of high-velocity PSRs is far-reaching; it has an impact on the mechanisms that give rise to PSR velocities at birth, and it affects how they interact with their surroundings and what the final distribution of PSRs in the Galaxy will be. If this result holds up we will need to re-examine the birth rate of PSRs, their survival in binary systems and their escape from globular clusters and the Galaxy (Lyne \& Lorimer 1994). Future efforts should concentrate on measuring proper motions to test the veracity of the associations. Both the magnitude and the direction of the velocity vector are useful in this regard. The former will test whether the high velocities are real and the latter will test the association (i.e., the PSR must originate from the remnant).

If a significant population of high-velocity PSRs does exist then they could escape the disk, forming a halo population of old neutron stars. This extended halo population has been postulated to exist for many years in order to explain $\gamma$-ray bursts as a galactic phenomenon (e.g., Li \& Dermer 1992 ) but critics have argued that an "ad hoc" population of Galactic objects is less preferable to a cosmological distribution of sources (Paczyński 1993). Now that evidence is accumulating in favor of a real high-velocity neutron star population and it has been shown that they are able to reproduce the high degree of angular isotropy seen by current $\gamma$-ray instruments (Podsiadlowski, Rees \& Ruderman 1994) there is cause to revisit the extended halo models. To produce the observed distribution of $\gamma$-ray bursts the high-velocity PSRs must be endowed with a special property that distinguish them from the low-velocity PSRs, and this may be related to the origin of the bursts. High magnetic fields $\left(10^{14}-10^{15} \mathrm{G}\right)$ have been evoked in some models of bursters (e.g., Duncan \& Thompson 1992) but the dipole fields of the PSRs in the table are only slightly above the mean $\log B$ of 12.51 (Stollman 1987).

With $V_{\mathrm{PSR}} \simeq 500 \mathrm{~km} \mathrm{~s}^{-1}$ a PSR catches up to its SNR in only $40000-$ 70000 years (Shull et al. 1989). Such PSRs will act as a "fountain of youth" injecting fresh relativistic particles and field into the compressed shell of the aging remnant. Thus ambient density may not be the dominant factor influencing the radio lifetimes of supernova remnants. The distinction between shell-type remnants and pulsar-powered nebulae has been blurred, creating a new class of supernova remnants called "interacting composites". Examples may include a number of objects mentioned by Shull et al. (1989) like G 5.4-1.2, W 28 and G 57.1+1.7 as well as G $114.3+0.3$ (Kulkarni et al. 1993), G308.8-0.1 (Kaspi et al. 1992), and MSH 15-52 (Caswell, Milne \& Wellington 1981). 


\section{References}

Allakhverdiyev, A.O., Amnuel, P.R., Guseinov, O.H. \& Kasumov, F.K. 1983, ApSS 97, 261

Aschenbach, B. 1992, in Highlights of Astronomy, Vol. 9, J. Bergeron (Ed.), Kluwer (Dordrecht), p. 223

Bailes, M. \& Johnston, S. 1993 in Review of Radio Science (URSI), W. Ross Stone (Ed.), Oxford University Press, London, p. 677

Bailes, M. et al. 1989, ApJ 343, L53

Blandford, R., Applegate, J.H. \& Hernquist, L. 1983, MNRAS 204, 1025

Caraveo, P.A. 1993, ApJ 415, L111

Caswell, J.L., Milne, D.K. \& Wellington, K.J. 1981, MNRAS 195, 89

Caswell, J.L. et al. 1992, ApJ 399, L151

Chevalier, R.A. 1994, in IAU Coll. 145, R. McCray \& Z. Wang (Eds.), Cambridge U. Press (London), (in press)

Clark, D.H. \& Caswell, J.L. 1976, MNRAS 174, 267

Cordes, J.M. 1986, ApJ 311, 183

Cornwell, T.J. 1993, VLA Scientific Memorandum 164, National Radio Astronomy Observatory

Duncan, R.C. \& Thompson, C. 1992, ApJ 392, L9

Dwarakanath, K.S. 1991, J. Astrophys. Astr. 12, 199

Frail, D.A. \& Kulkarni, S.R. 1991, Nat 352, 785

Frail, D.A. \& Moffett, D.A. 1993, ApJ 408, 637

Frail, D.A., Goss, W.M. \& Whiteoak, J.B.Z. 1994, ApJ (in press)

Harrison, P.A., Lyne, A.G. \& Anderson B. 1993, MNRAS 261, 113

Helfand, D.J. \& Becker, R.H. 1984, Nat 307, 215

Helfand, D.J. \& Tademaru, E. 1977, ApJ 216, 842

Johnston, S. et al. 1992, MNRAS 255, 401

Johnston, S. et al. 1994, A\&A (in press)

Kaspi, V. M. et al. 1992, ApJ 399, L155

Kassim, N.E. \& Weiler, K.W. 1990, Nat 343, 146

Kulkarni, S.R. 1990, in Neutron Stars and Their Birth Events, W. Kundt (Ed.), Kluwer Academic Publishers, p. 59

Kulkarni, S.R., Predehl, P., Hasinger, G. \& Aschenbach, B. 1993, Nat 362, 135

Li, H. \& Dermer, C.D. 1992, Nat 359, 514

Lyne, A.G. \& Lorimer D.R. 1994, Nat 369, 127

Manchester, R.N., D'Amico, N. \& Tuohy, I.R. 1985, MNRAS 212, 975

Manchester, R.N. et al. 1991, MNRAS 253, P7

McAdam, W.B., Osborne, J.L. \& Parkinson, M.L. 1993, Nat 361, 516

Milne, D.K. 1979, Aus. J. Phys. 32, 83

Narayan, R. 1987, ApJ 319, 162

Narayan, R. \& Schaudt, K.J. 1988, ApJ 325, L43

Paczyński, B. 1993, in Compton Gamma Ray Observatory, M. Friedlander, N. Gehrels \& D.J. Macomb (Eds.), AIP (New York), p. 981

Podsiadlowski, P., Rees, M.J. \& Ruderman, M. 1994, MNRAS (preprint)

Shull, J.M., Fesen, R.A. \& Saken, J.M. 1989, ApJ 346, 860

Stollman, G.M. 1987, A\&A 178, 143

Taylor, J.H. \& Cordes, J.M. 1993 ApJ, 411, 674

Taylor, A.R., Wallace, B.J. \& Goss, W.M. 1992, AJ 103, 931 